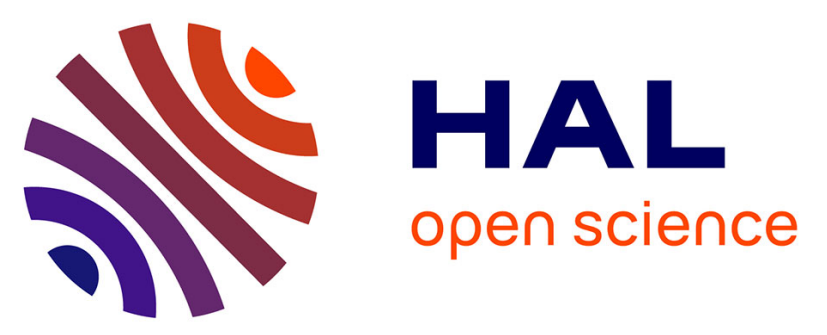

\title{
Propagation des ondes élastiques de surface - Atlas des configurations calculées pour le quartz, le tantalate de lithium, le niobate de lithium et le vanado-sulfure de thallium en ce qui concerne les coefficients de température et le couplage piézo-électrique
}

\author{
M. Feldmann, J. Henaff
}

\section{To cite this version:}

M. Feldmann, J. Henaff. Propagation des ondes élastiques de surface - Atlas des configurations calculées pour le quartz, le tantalate de lithium, le niobate de lithium et le vanado-sulfure de thallium en ce qui concerne les coefficients de température et le couplage piézo-électrique. Revue de Physique Appliquée, 1977, 12 (11), pp.1775-1788. 10.1051/rphysap:0197700120110177500 . jpa-00244402

HAL Id: jpa-00244402

https://hal.science/jpa-00244402

Submitted on 1 Jan 1977

HAL is a multi-disciplinary open access archive for the deposit and dissemination of scientific research documents, whether they are published or not. The documents may come from teaching and research institutions in France or abroad, or from public or private research centers.
L'archive ouverte pluridisciplinaire HAL, est destinée au dépôt et à la diffusion de documents scientifiques de niveau recherche, publiés ou non, émanant des établissements d'enseignement et de recherche français ou étrangers, des laboratoires publics ou privés. 


\title{
PROPAGATION DES ONDES ELASTIQUES DE SURFACE
}

\author{
Atlas des configurations calculées pour le quartz, le tantalate de lithium, \\ le niobate de lithium et le vanado-sulfure de thallium \\ en ce qui concerne les coefficients de température et le couplage piézo-électrique
}

\author{
M. FELDMANN et J. HENAFF \\ Centre National d'Etudes des Télécommunications \\ Département TCR/DEF, 92131 Issy-les-Moulineaux, France
}

(Reçu le 2 mai 1977, révisé le 25 juillet 1977, accepté le 26 juillet 1977)

\begin{abstract}
Résumé. - La tenue en température est souvent une exigence très contraignante pour les dispositifs à ondes élastiques de surface destinés à être insérés dans les systèmes. Cet article explore à cet égard les performances de l'ensemble des configurations possibles des matériaux classiques (quartz, niobate de lithium et tantalate de lithium) ainsi que d'un matériau récemment signalé le vanado-sulfure de thallium $\left(\mathrm{Tl}_{3} \mathrm{VS}_{4}\right)$. La propagation des ondes de surface est définie par la donnée du plan de la coupe et d'une direction dans ce plan, c'est-à-dire par trois paramètres à une température fixée : ce grand nombre de paramètres explique le fait que toutes les configurations ne soient pas encore complètement explorées. Les résultats sont rassemblés sous forme de cartes donnant les couplages piézo-électriques et les coefficients de température. Ces cartes permettent de sélectionner un certain nombre de configurations possédant à la fois un couplage élevé et une bonne stabilité en température. Enfin, quelques configurations sélectionnées sont décrites en détail.
\end{abstract}

\begin{abstract}
The temperature behaviour is often an essential feature in acoustic surface wave device applications. This paper gives a set of computed results regarding conventional materials (quartz, lithium niobate and lithium tantalate) and also a new promising one, the $\mathrm{Tl}_{3} \mathrm{VS}_{4}$. Since acoustic - surface - wave propagation is characterized by both cut and propagation direction, the set of configurations depends upon three parameters at a given temperature : this rather large number of parameters explains why these configurations have not been yet completely described. The results are collected in an atlas of coupling and temperature coefficient maps. From these maps it is possible to select some interesting configurations which combine both high

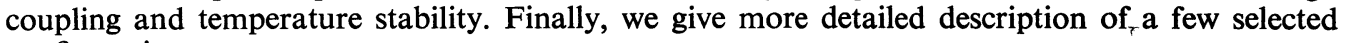
configurations.
\end{abstract}

1. Introduction. - Les dispositifs à ondes élastiques de surface se sont révélés particulièrement utiles, dans la réalisation de filtres, d'oscillateurs, de lignes à retard. La plupart de ces dispositifs utilisent comme matériaux le quartz, le niobate de lithium $\left(\mathrm{LiNbO}_{3}\right)$ et aussi le tantalate de lithium $\left(\mathrm{LiTaO}_{3}\right)$. La situation dans ce domaine peut être résumée de la façon suivante : il existe de bons matériaux piézo-électriques, c'est-à-dire à fort couplage électromécanique, comme le tantalate de lithium et surtout le niobate de lithium mais dont les coupes connues, sont affectées d'une forte sensibilité à la température (de l'ordre de $90 \mathrm{ppm} / \mathrm{K}$ ) ce qui rend parfois les dispositifs inaptes à remplir leur fonction.

Il existe un matériau à couplage médiocre, le quartz, qui bénéficie d'une coupe à coefficient de température au premi er ordre nul, la coupe ST.
Récemment [1, 3], il a été démontré qu'un matériau cubique, le $\mathrm{Tl}_{3} \mathrm{VS}_{4}$ semble associer un fort couplage piézo-électrique (quelques \%). et des coupes à coefficient de température en fréquence nul.

Cet article se propose de faire le point sur les quatre matériaux cités grâce à un calcul assez exhaustif pour l'ensemble des configurations possibles, des coefficients de couplage piézo-électrique et des coefficients de température en fréquence (CTF).

2. Programme de calcul de nouvelles coupes. 2.1 Principe. - Il n'y a guère de guide sûr pour chercher des coupes à coefficient de température nul. On peut toutefois affirmer que le coefficient de température ne peut s'annuler que s'il existe des coefficients de température des deux signes en ondes de volume. Ce critère élimine le niobate de lithium parmi des 
candidats possibles. Pour le reste, la tabulation des coefficients de température semble le seul moyen utilisable. Or, en ondes de surface, le nombre de points à explorer (couple : coupe et direction de propagation) est très grand: pour un pas de $5^{\circ}$, il existe 1650 directions sur la sphère et 72 directions sur le cercle soit 118800 calculs pour une exploration à température donnée. Un coefficient de température exigeant deux calculs différents, chacun durant environ $0,1 \mathrm{mn}$ (sur l'ordinateur HB 6000) on voit qu'une tabulation complète exigerait environ 10 jours de calcul !

Heureusement, on peut tenir compte des symétries et utiliser un pas plus grossier, sauf dans les zones ambiguës. L'examen d'un matériau donné comme le quartz n'en nécessite pas moins une dizaine d'heures de calcul sans compter le temps de mise au point des programmes.

2.2 Calcul. - Le principe du calcul lui-même ne présente pas d'originalité particulière. L'organigramme utilisé (Fig. 1) est voisin de celui utilisé par Campbell et Jones en 1968 [4].

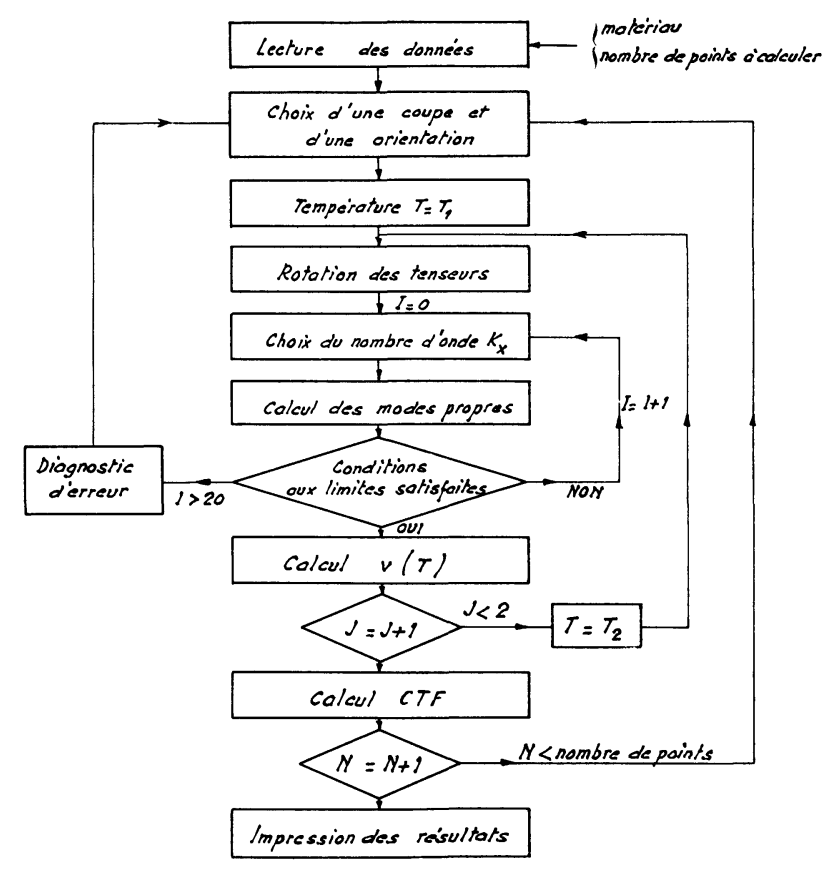

FIG. 1. - Organigramme du calcul du CTF (coefficient de température en fréquence).

[FTC (frequency temperature coefficient) process chart of computer program.]

Les constantes des matériaux, ont été prises dans la littérature et sont données en annexes en ce qui concerne le quartz (I), le tantalate de lithium (II), le niobate de lithium (III) et le vanado-sulfure de thallium (IV).

Le calcul se conduit de la façon suivante: on choisit une tabulation adéquate avec un pas assez grossier $\left(10^{\circ}\right)$, tenant compte de la symétrie rhombo- édrique ou cubique des matériaux étudiés, et de la dégénérescence de Kramer dans les vitesses... Pour chaque point à tabuler, on fixe une température de référence $\left(20^{\circ} \mathrm{C}\right)$ et on calcule pour deux températures $T-\Delta T$ et $T+\Delta T$ les vitesses de phase. Pour cela, on exprime les tenseurs des constantes dans des axes canoniques liés à la coupe, et on calcule les 4 modes propres ayant une composante fixée $K_{x}$ du vecteur d'onde à la fréquence unité et dans le plan de la coupe.

A cette pulsation unité, la vitesse $v$ est identifiée à $1 / K_{x}$. En définitive, la combinaison des quatre modes doit permettre de satisfaire aux conditions aux limites tant mécaniques qu'électriques à la surface du cristal. La prise en compte des seules conditions mécaniques définit une relation entre les grandeurs électriques pouvant se mettre sous la forme

où

$$
\frac{D_{z}}{E_{x}}=\varepsilon_{\mathrm{s}}(v)
$$

- $\varepsilon_{\mathrm{s}}(v)$, fonction de $v=1 / K_{x}$, est la permittivité de surface du milieu,

- $D_{z}$ est le déplacement électrique normal, et

- $E_{x}$ est le champ électrique.

On recherche le zéro $v_{0}$ et le pôle $v_{\infty}$ de $1 / \varepsilon_{\mathrm{s}}(v)$ correspondant respectivement, aux conditions aux limites électriques d'un milieu extérieur conducteur et d'un milieu extérieur de permittivité nulle, par itération sur la valeur de $K_{x}$. Le coefficient de couplage lié au résidu de $1 / \varepsilon_{\mathrm{s}}(v)$ au pôle $v_{\infty}$ est voisin de $\left(v_{\infty}^{2}-v_{0}^{2}\right) / v_{\infty}^{2}$ (la théorie correspondante est explicitée dans la référence [5]).

Simultanément, on calcule le coefficient de dilatation dans la direction de propagation, ce qui conduit à une constante de fréquence, dont la variation relative entre $T-\Delta T$ et $T+\Delta T$ donne le CTF cherché pour des conditions électriques définies par la permittivité du milieu extérieur. L'atlas ne reproduit toutefois que le CTF correspondant à la vitesse $v_{\infty}$.

2.3 PRÉSENTATION DES RÉSUltats. - Les résultats sont résumés par la ligne de CTF de niveau zéro (lorsqu'il existe), dans la projection stéréographique des normales aux différentes coupes. En réalité, chaque projection stéréographique est associée à une direction de propagation donnée dans la coupe, par rapport à une référence qui est ici prise à l'intersection du plan de la coupe et du plan $X O Y$ (Fig. 2). (On prend $O X$ comme référence dans la direction de coupe $Z$.)

Finalement, la représentation d'un couple coupedirection de propagation nécessite trois paramètres réels et l'ensemble des points peut être figuré par un volume à trois dimensions. Les trois paramètres étant des angles, leur périodicité permet d'imaginer ce volume comme un tore dont la section transversale représente la projection stéréographique (Fig. 3) 


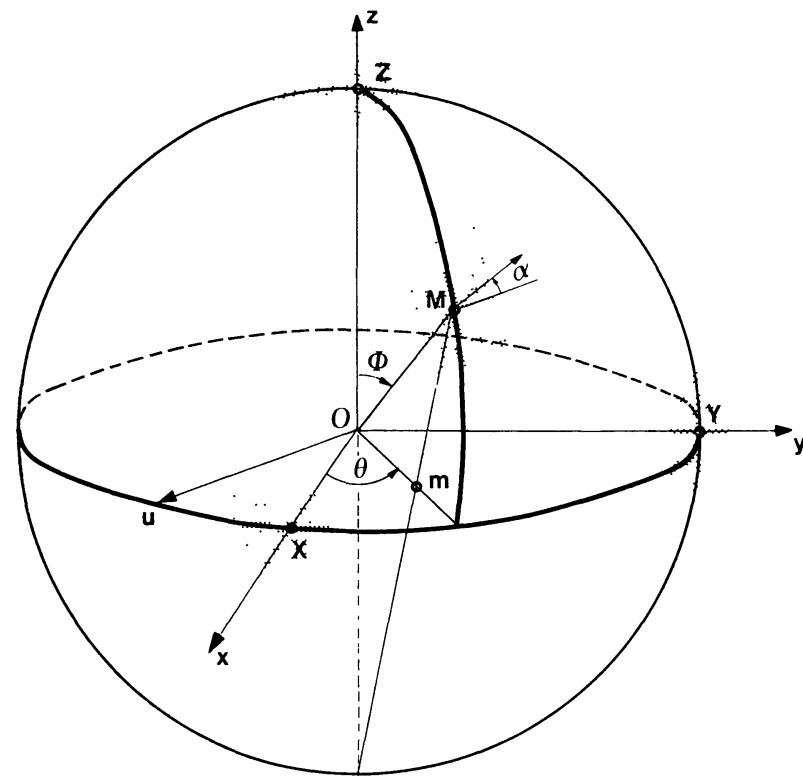

Fig. 2. - Repérage de la coupe et de l'orientation. La normale au plan est repérée par les angles $\theta$ et $\varphi$. La direction de propagation dans le plan est repérée par rapport à l'intersection Ou de ce plan avec le plan $(x y)$.

[Characteristic angles $(\alpha, \theta, \varphi), \theta$ and $\varphi$ reference the normal to the cut-plane and $\alpha$ is the angle between the direction of propagation and the intersecion Ou of this plane with the $(x y)$ plane.]

des coupes et dont les parallèles correspondent aux directions de propagation dans chaque coupe.

Quoi qu'il en soit, à l'intérieur de ce volume torique, on recherche les nappes d'iso-CTF et d'iso-couplage.

2.4 Résultats CONCERnant Le QUaRTz. - Dans le tore représentatif des configurations, on note qu'il existe une nappe de CTF nul passant comme il se doit par la configuration dite ST. Cette nappe est

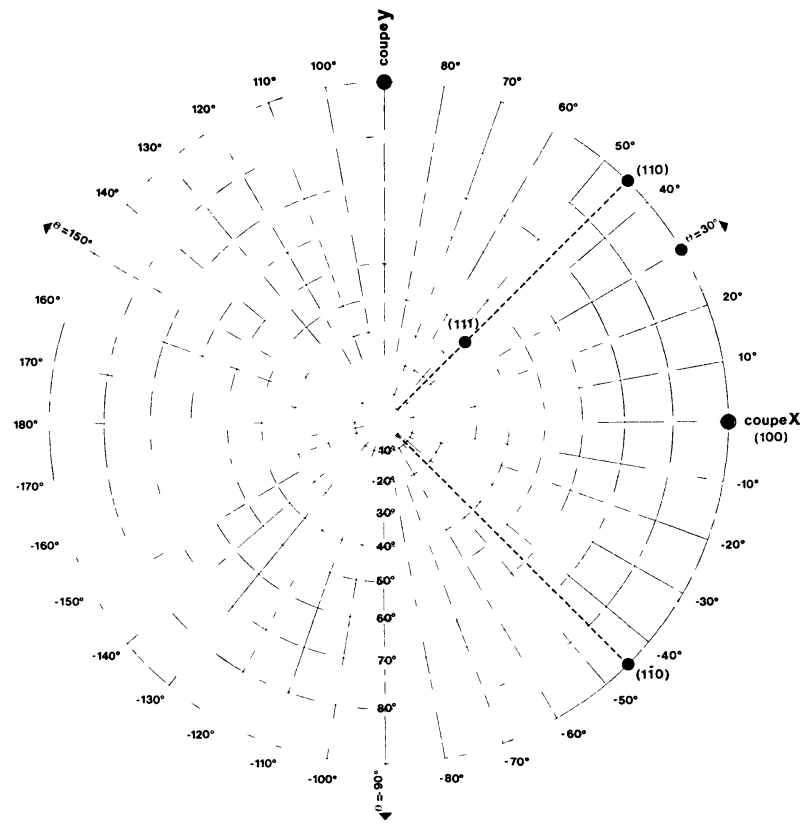

Fig. 3. - Projection stéréograp'ique repérant les coupes.

[Stereographic projection referencing the cuts.]

représentée dans 10 sections droites du tore (Figs. $4 a-4 j$ ). Dans le système rhomboédrique, chaque section droite admet un axe de répétition d'ordre 3. Grâce à la symétrie de Kramer, lcs orientations correspondant à des directions de propagation $\alpha$ et $\pi+\alpha$ redonnent la même valeur. En outre, le produit de la symétrie autour de l'axe $O Y$ et de la symétrie de Kramer entraîne que les sections droites associées aux angles $\alpha$ et $-\alpha$ sont elles-mêmes symétriques l'une de l'autre, ce qui permet de rétablir l'ensemble des configurations. La nappe à CTF nul doit être étudiée en fonction du coefficient de couplage $k^{2}$. On a porté sur les projections stéréographiques les lignes d'iso-couplage

\section{TABLEAU I}

Paramètres principaux des coupes intéressantes dans le quartz, le $\mathrm{LiTaO}_{3}$, le $\mathrm{LiNbO}_{3}$ [Main parameters of significant orientations in quartz, $\mathrm{LiTaO}_{3}, \mathrm{LiNbO}_{3}$ ]

\begin{tabular}{|c|c|c|c|c|c|c|c|c|c|c|c|c|c|c|c|}
\hline & \multicolumn{3}{|c|}{ Angles } & \multicolumn{3}{|c|}{ Coupe } & \multicolumn{3}{|c|}{ Propagation } & \multirow{2}{*}{$\frac{v}{\mathrm{~m} / \mathrm{s}}$} & \multirow{2}{*}{$\frac{k^{2}}{\%}$} & \multirow{2}{*}{$\frac{\Psi\left(^{b}\right)}{d^{o}}$} & \multirow{2}{*}{$\frac{\beta(c)}{10^{-8} \mathrm{~K}^{-2}}$} & \multirow{2}{*}{$\frac{\mathrm{CTF}}{\mathrm{ppm}} \mid$} & \multirow[t]{2}{*}{$\varepsilon_{\mathrm{LF}} / \varepsilon_{0}$} \\
\hline & $\alpha$ & $\theta$ & $\varphi$ & $X$ & $Y$ & $Z$ & $X$ & $Y$ & $Z$ & & & & & & \\
\hline Quartz : ST ( $\left.{ }^{a}\right)$ & $0^{\circ}$ & $90^{\circ}$ & $47^{\circ} 25$ & 0 & 0,734 & 0,679 & -1 & 0 & 0 & $\begin{array}{ll}3 & 159,2\end{array}$ & 0,14 & 0 & -4 & 0 & 4,56 \\
\hline $\begin{array}{l}\text { Quartz: } \\
k^{2} \max \text { à } C T F=0\end{array}$ & $20^{\circ}$ & $45^{\circ}$ & $45^{\circ}$ & 0,500 & 0,500 & 0,707 & $-0,835$ & 0,493 & 0,242 & 3493,9 & 0,17 & $3^{\circ} 86$ & $-8,7$ & 0 & 4,56 \\
\hline $\mathrm{LiTaO}_{3}:(\mathrm{A})$ & $90^{\circ}$ & $\overline{30^{\circ}}$ & $80^{\circ}$ & $\overline{0,853}$ & $\overline{0,492}$ & $\overline{0,174}$ & $-0,150$ & $-0,087$ & $\overline{0,985}$ & $3 \overline{298,6}$ & $\overline{1,36}$ & 0 & - & 31 & 47,29 \\
\hline $\mathrm{LiTaO}_{3}:(\mathrm{B})$ & $20^{\circ}$ & $25^{\circ}$ & $35^{\circ}$ & $\overline{0,520}$ & $\overline{0,242}$ & $\overline{0,819}$ & $-0,651$ & 0,733 & $\overline{0,196}$ & $\overline{3423,8}$ & 1,52 & 1033 & - & 49 & 48,25 \\
\hline $\mathrm{LiNbO}_{3}: Y Z$ & $90^{\circ}$ & $90^{\circ}$ & $90^{\circ}$ & 0 & 1 & 0 & 0 & 0 & 1 & 3491,1 & 4,86 & 0 & - & 94 & 45,84 \\
\hline $\mathrm{LiNbO}_{3}:$ [réf. 7] & $0^{\circ}$ & $30^{\circ}$ & $41^{\circ} 5$ & $\overline{0,574}$ & $\overline{0,331}$ & $\overline{0,749}$ & $-0,500$ & 0,866 & 0 & 4003,6 & 5,57 & 0 & - & 72 & 55,19 \\
\hline
\end{tabular}

(a) Référence : Schulz, M. B., Matsinger, B. J. et Holland, M. G. : « Temperature dependence of surface acoustic wave velocity on alpha-quartz », J. Appl. Phys. 41 (1970) 2755.

(b) $\Psi$, angle de dérive (" power flow angle ").

$\left.{ }^{(}\right) \beta$, coefficient de température du deuxième ordre. 

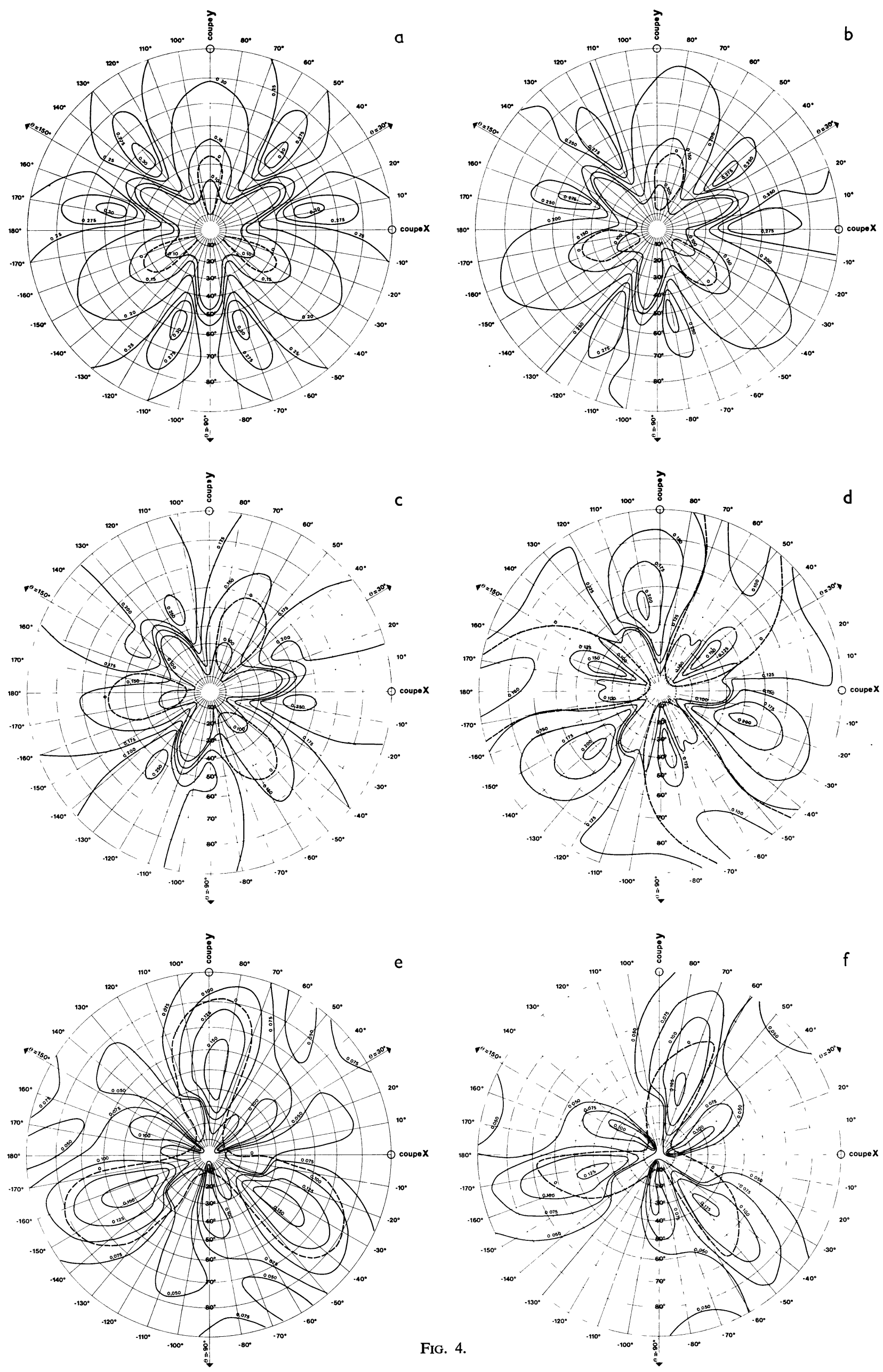

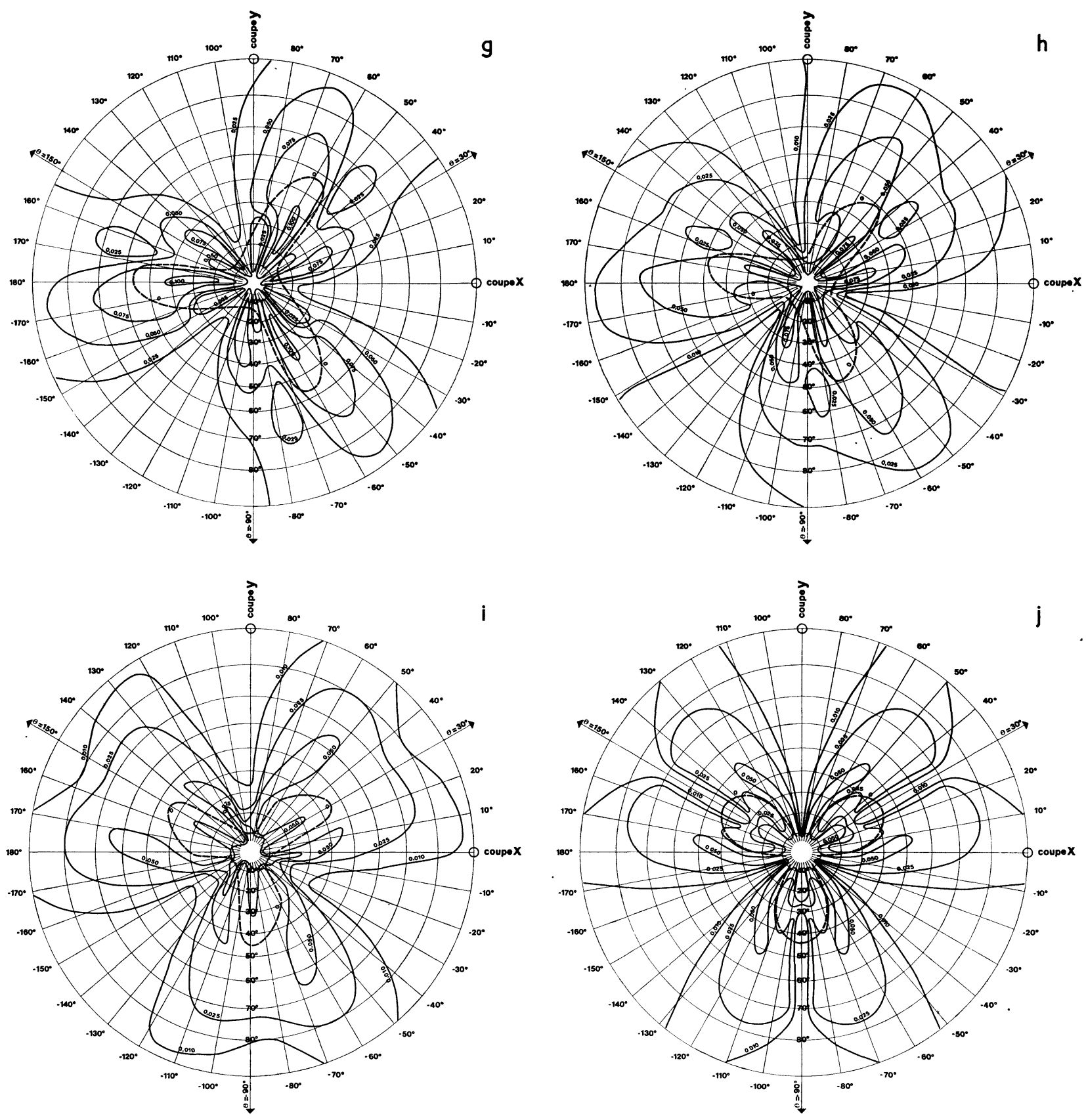

FIG. 4. - Carte des lignes d'iso-couplage et d'iso-CTF correspondant aux orientations $\alpha=0^{\circ}(4 a)$ à $\alpha=90^{\circ}(4 j) \mathrm{du}$ quartz. — en trait plein : coefficients de couplage exprimés en $\%, \ldots$ - en tireté : coefficients de température en fréquence (CTF) égal à zéro.

[Map of equal $-k^{2}$ and equal - FTC corresponding to the orientations $\alpha=0^{\circ}(4 a)$ to $\alpha=90^{\circ}$ ( $4 j$ ) for quartz. Solid lines : $k^{2}$ in $\%$. Broken line : FTC $=0$.] 
correspondant à $k^{2}=0,1 \%$ à $k^{2}=0,3 \%$ par pas de $0,05 \%$.

On relève que les points de plus fort couplage $\left(k^{2}>0,3 \%\right)$ sont rassemblés dans les sections $\alpha=0$ et $\alpha=10^{\circ}$. Malheureusement ces « forts " couplages ne correspondent pas à un CTF nul. La coupe ST avec un couplage de $0,13 \%$ reste l'une des plus favorables. (Notons que la valeur du couplage donnée par la littérature est de $0,16 \%$ pour cette coupe. La différence de $20 \%$ provient d'une définition elle-même légèrement différente et explicitée dans la référence [5].)

Toutefois, l'optimum est une coupe doublement tournée correspondant à $\alpha=20^{\circ}, \theta=45^{\circ}$ et $\varphi=45^{\circ}$ avec un couplage de $30 \%$ plus fort que celui de la coupe ST. Cet avantage, peut dans certains cas compenser la complexité de découpe des lames cristallines correspondantes. Les paramètres caractéristiques de ces 2 coupes sont résumés dans le tableau I.

2.5 RÉSUltats CONCERNANT LE TANTAlate DE LITHIUM. - La même procédure a été appliquée au tantalate de lithium. On note d'abord qu'il n'existe aucune orientation à CTF nul (Figs. $5 a-5 j$ ), les coefficients de température s'étageant de 30 à 60 ppm environ.

Les coefficients de couplage piézo-électrique sont définis avec la même convention que pour le quartz. En outre il existe une incertitude beaucoup plus grande sur la valeur des coefficients $e_{i j}$ de la matrice de couplage, incertitude liée notamment à l'influence de la stœchiométrie $\mathrm{Li} / \mathrm{Ta}$ dans les échantillons commerciaux.

Avec ces réserves, on relève parmi les zones les plus favorables une coupe $\mathrm{Y}$ tournée $\left(\alpha=90^{\circ}\right.$, $\theta=30^{\circ}$ et $\varphi=80^{\circ}$ ) avec un couplage de $1,4 \%$ et un CTF de $30 \mathrm{ppm}$. Le couplage maximal dépasse $1,5 \%$ pour une coupe doublement tournée $\left(\alpha=20^{\circ}\right.$, $\theta=25^{\circ}$ et $\varphi=35^{\circ}$ ) (cf. Tableau I).

Enfin, on a contrôlé expérimentalement la conformité des données pour les trois configurations $Y Z$, $Z Y$ et $X Z$. Les mesures ont été réalisées sur des lignes dont les caractéristiques principales sont les suivantes : longueur d'onde $26,26 \mu \mathrm{m}$, ouverture $2 \mathrm{~mm}, 91$ et 181 dents pour chaque peigne respectivement et un chemin acoustique de 90 longueurs d'onde.

Les valeurs expérimentales de la vitesse, du couplage et du CTF mesurés dans un montage en oscillateur [8, 9] sont résumées dans le tableau II.

Comparé aux valeurs théoriques, on note un accord remarquable en ce qui concerne le CTF, convenable en ce qui concerne la vitesse mais assez médiocre pour le couplage.

En outre on note une différence avec les résultats théoriques publiés par Schulz et Holland [6] utilisant apparemment les mêmes constantes.

2.6 RÉSULTATS CONCERNANT LE NIOBATE DE LITHIUM. - Le niobate de lithium n'a pas a priori de coupe

\section{TABLEAU II}

Résumé des caractéristiques des 3 configurations classiques du $\mathrm{LiTaO}_{3}$

[Characteristics of the 3 conventional configurations of lithium tantalate]

$\begin{array}{cccc} & X Z & Z Y & Y Z \\ & \theta=120^{\circ}, & \theta_{\text {ind. }}, & \theta=90^{\circ}, \\ & \varphi=90^{\circ}, & \varphi=0^{\circ}, & \varphi=90^{\circ}, \\ & \alpha=90^{\circ} & \alpha=90^{\circ} & \alpha=90^{\circ} \\ & - & - & - \\ \text { rès Schulz } & & & \\ \text { Holland [6] } & & & \\ k^{2} & 0,64 \% & 1 \% & 0,97 \% \\ \text { CTF } & 35 \mathrm{ppm} & 66 \mathrm{ppm} & 35 \mathrm{ppm}\end{array}$

D'après Schulz

et Holland [6]

D'après le pro-

gramme de cal-

cul du CNET

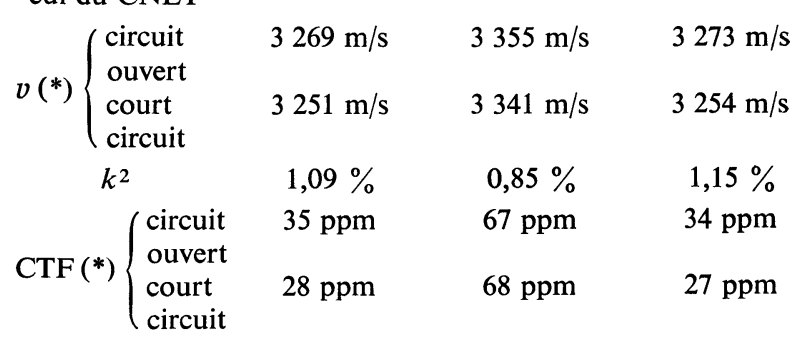

D'après les mesures effectuées au CNET

$\begin{array}{cccc}v & 3230 \mathrm{~m} / \mathrm{s} & 3343 \mathrm{~m} / \mathrm{s} & 3240 \mathrm{~m} / \mathrm{s} \\ k^{2} & 0,83 \% & 1,3 \% & 0,88 \% \\ \text { CTF } & 30 \mathrm{ppm} & 73 \mathrm{ppm} & 31 \mathrm{ppm}\end{array}$

(*) Le calcul donne deux valeurs de vitesse et de CTF correspondant à une surface libre ou court-circuitée. L'atlas ne reproduit que celle du vide (la plus défavorable d'ailleurs).

à coefficient de température nul. C'est bien ce que vérifie le calcul, l'atlas des configurations étant représenté sur les figures $6 a-6 j$. Le CTF est même assez constant à des valeurs élevées de l'ordre de 75-95 ppm.

En ce qui concerne le couplage piézo-électrique, on note que le plus fort couplage atteint $5,5 \%$, à comparer avec le couplage de $4,45 \%$ de la coupe $Y Z$ classique. L'orientation correspondante $(\alpha=0$, $\theta=30^{\circ}$ et $\varphi=40^{\circ}$ ) admet en outre un CTF faible (72 ppm) : il s'agit d'une coupe $Y$ simplement tournée de $130^{\circ}$. Cette coupe pour $\varphi=41^{\circ} 5$ a déjà été signalée par Slobodnik et Conway [7] (cf. Tableau I).

2.7 RÉSUltats CONCERNANT LE $\mathrm{Tl}_{3} \mathrm{VS}_{4}$. - L'atlas des configurations est représenté sur les figures $7 a$ à $7 j$.

On note que ce matériau admet un coefficient de couplage excellent, culminant à $3 \%$ environ ce qui le place entre le tantalate de lithium $(1,5 \%)$ et le niobate de lithium $(5,5 \%)$. En outre il présente une nappe à coefficient de température nul qui traverse à plusieurs reprises les zones à haut couplage. 

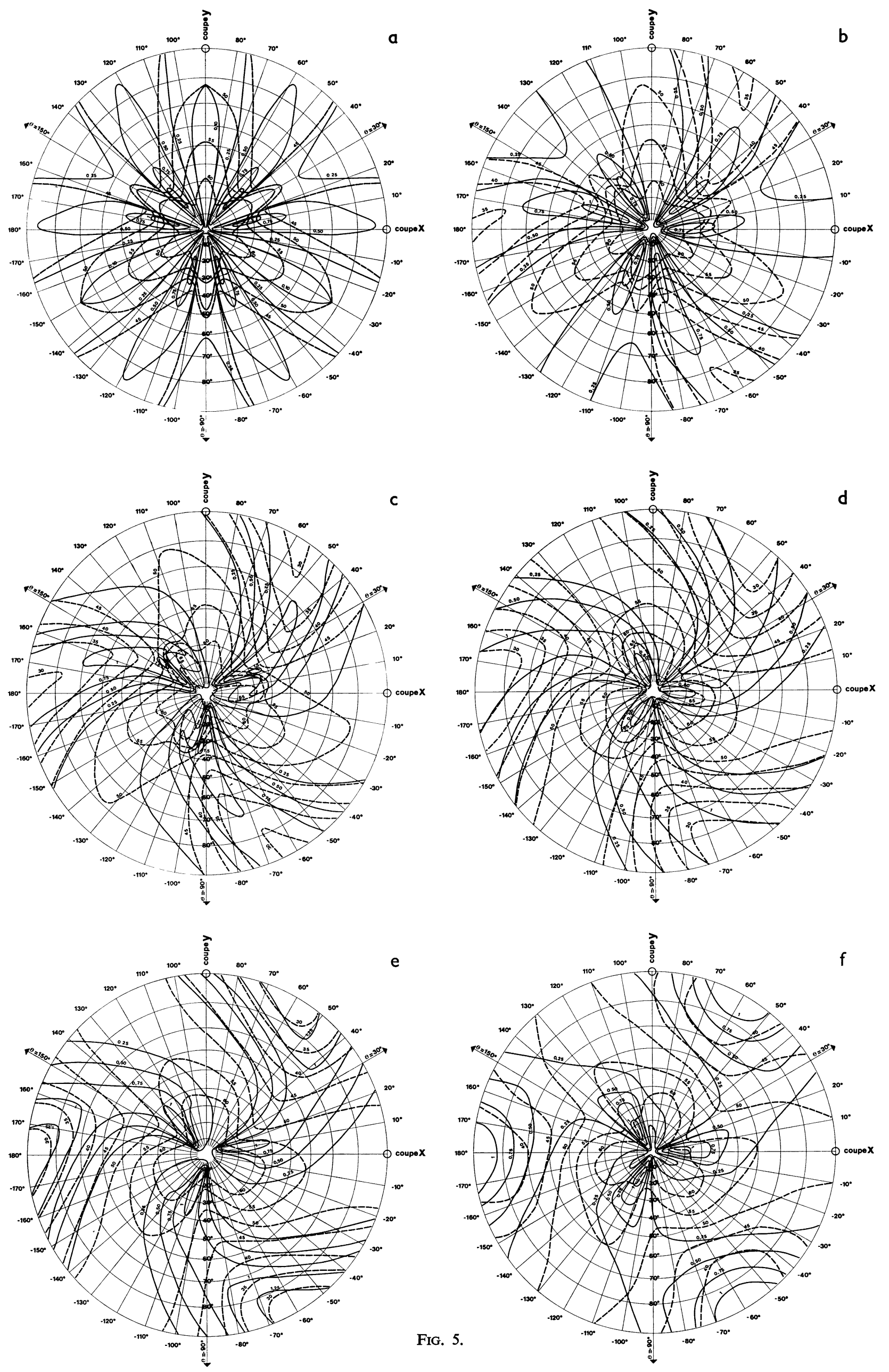

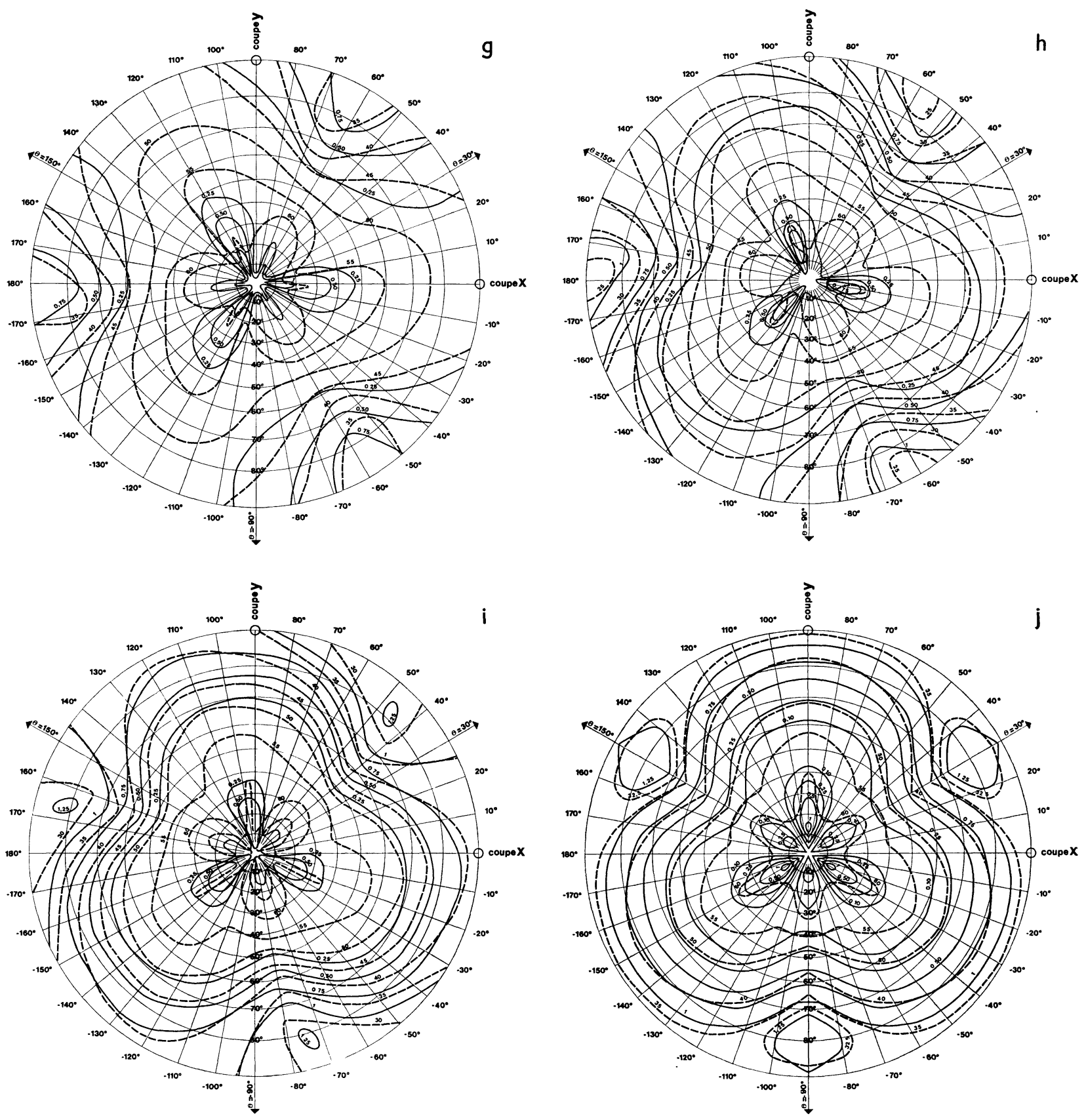

Fig. 5. - Carte des lignes d'iso-couplage et d'iso-CTF correspondant aux orientations $\alpha=0^{\circ}(5 a)$ à $\alpha=90^{\circ}(5 j) \mathrm{du}$ tantalate de lithium. — en trait plein : coefficients de couplage exprimé en $\%,-\cdots$ en tireté : CTF exprimés en ppm.

[Map of equal $-k^{2}$ and equal - FTC corresponding to the orientations $\alpha=0^{\circ}(5 a)$ to $\alpha=90^{\circ}(5 j)$ for lithium tantalate. Solid lines : $k^{2}$ in $\%$. Broken lines : FTC in ppm.] 

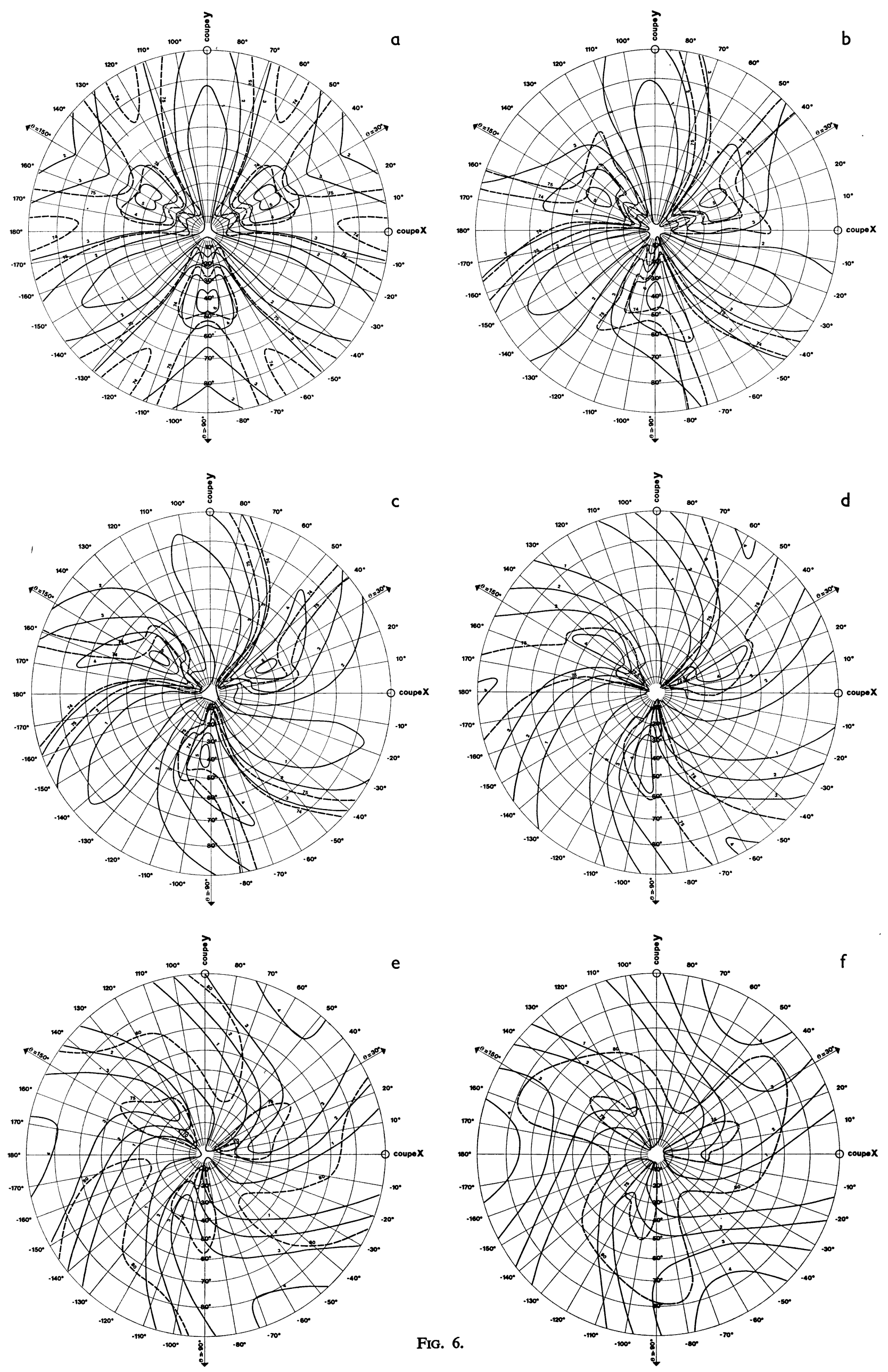

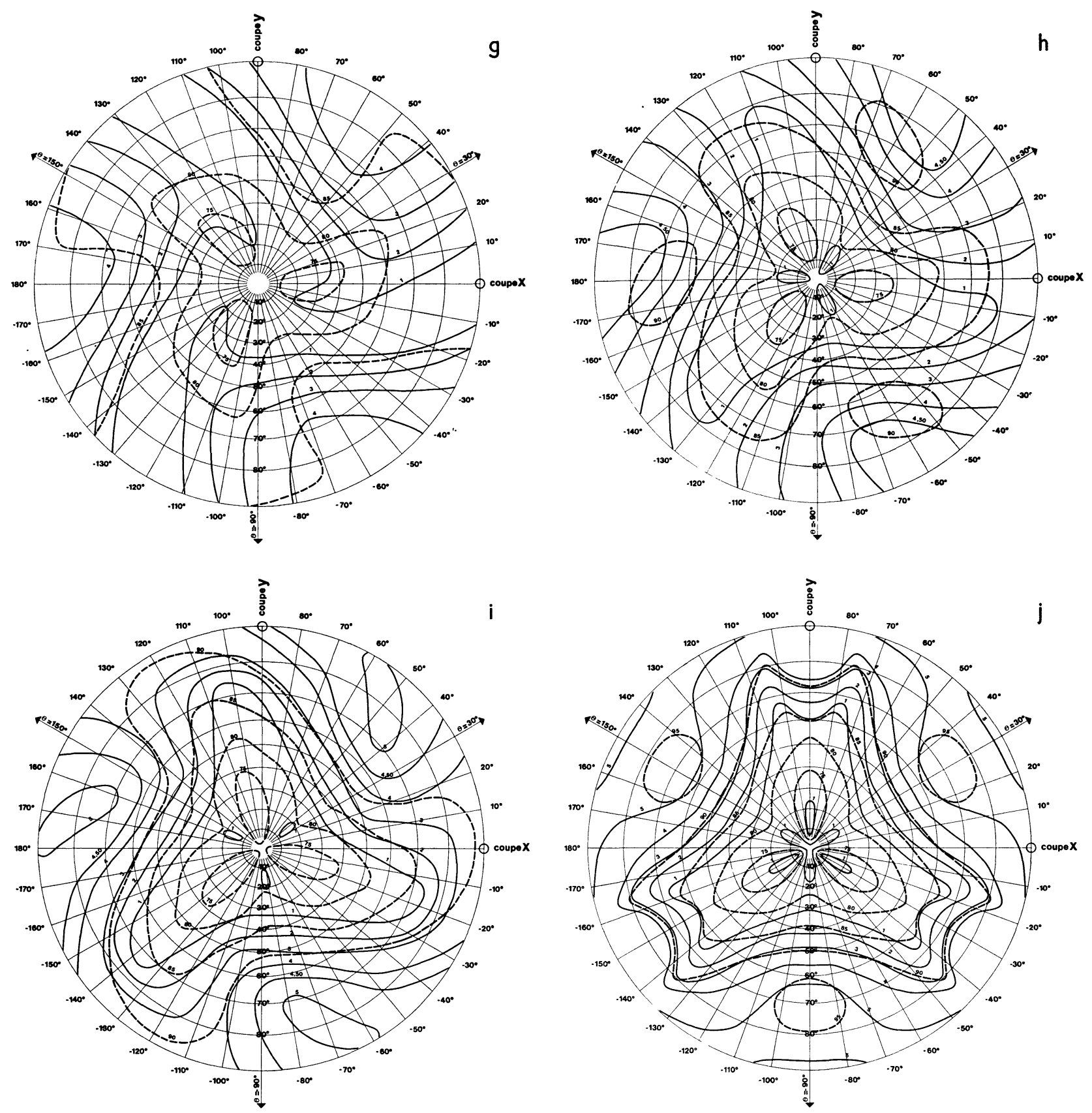

Fig. 6. - Carte des lignes d'iso-couplage et d'iso-CTF correspondant aux orientations $\alpha=0^{\circ}(6 a)$ à $\alpha=90^{\circ}(6 j)$ du niobate de lithium. — en trait plein : coefficients de couplage exprimés en \%, ---en tireté : CTF exprimés en ppm.

[Map of equal $-k^{2}$ and equal - FTC corresponding to the orientations $\alpha=0^{\circ}(6 a)$ to $\alpha=90^{\circ}(6 j)$ for lithium niobate. Solid lines : $k^{2}$ in $\%$. Broken lines : FTC in ppm.] 

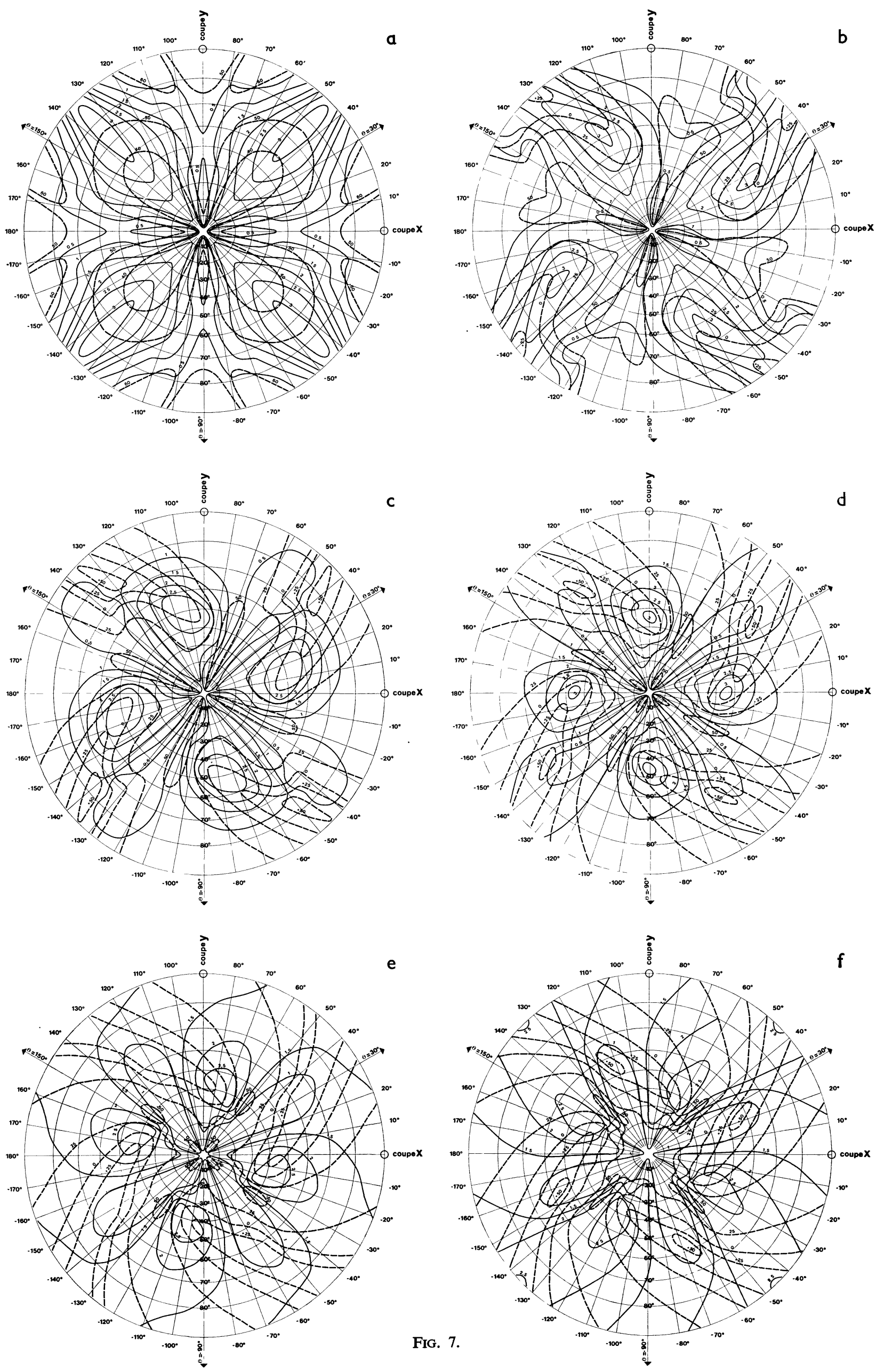

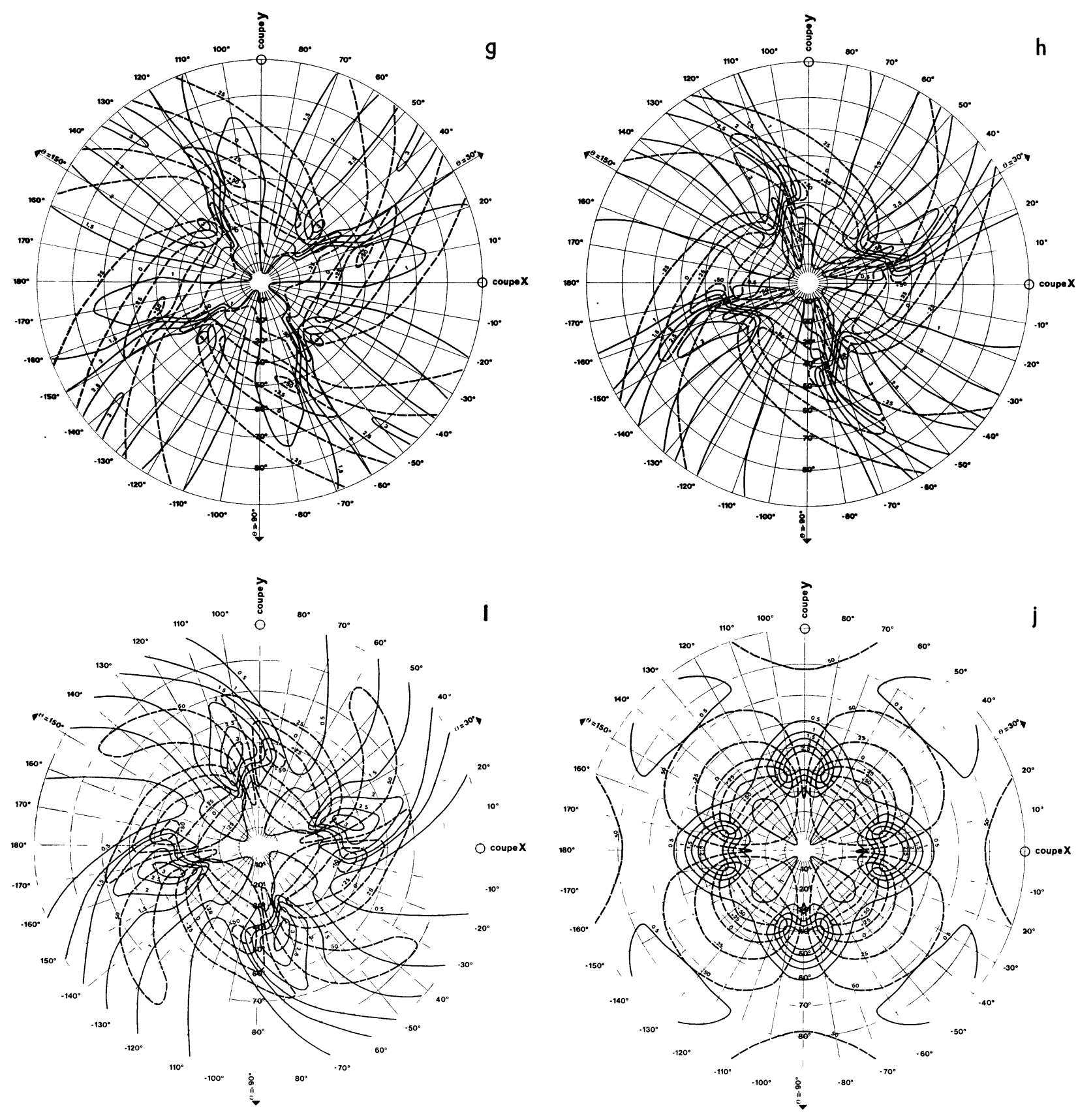

FIG. 7. - Carte des lignes d'iso-couplage et d'iso-CTF correspondant aux orientations $\alpha=0^{\circ}$ (7a) à $\alpha=90^{\circ}$ (7j) du vanado-sulfure de thallium. - en trait plein : coefficients de couplage exprimés en \%,-- - en tireté : CTF exprimés en ppm.

[Map of equal $-k^{2}$ and equal - FTC corresponding to the orientations $\alpha=0^{\circ}$ (7a) to $\alpha=90^{\circ}(7 j)$ for $\mathrm{Tl}_{3} \mathrm{VS}_{4}$. Solid lines : $k^{2}$ in $\%$. Broken lines : FTC in ppm.] 


\section{TABleAU III}

Paramètres principaux des coupes sélectionnées $d u \mathrm{Tl}_{3} \mathrm{VS}_{4}$ [Main parameters of selected cuts of $\mathrm{Tl}_{3} \mathrm{VS}_{4}$ ]

\begin{tabular}{|c|c|c|c|c|c|c|c|c|c|c|c|c|c|c|}
\hline & \multicolumn{3}{|c|}{ Angles } & \multicolumn{3}{|c|}{ Coupes } & \multicolumn{3}{|c|}{ Propagation } & \multicolumn{5}{|c|}{$\varepsilon_{\mathrm{BF} / \varepsilon_{0}}$} \\
\hline & $\alpha$ & $\theta$ & $\varphi$ & $X$ & $Y$ & $Z$ & $X$ & $Y$ & $Z$ & $v \mathrm{~m} / \mathrm{s}$ & $k^{2} \%$ & $(d)$ & $\psi\left({ }^{e}\right)$ & $\beta(d, f)$ \\
\hline & - & 一 & - & 一 & 一 & - & 一 & - & 一 & 二 & - & - & - & - \\
\hline $\mathbf{A}\left({ }^{d}\right)$ & $62^{\circ}$ & $45^{\circ}$ & $36^{\circ} 5$ & 0,421 & 0,421 & 0,804 & $-0,834$ & $-0,170$ & 0,525 & 976,9 & 3,02 & 15,27 & $17^{\circ} 8$ & -26 \\
\hline $\mathrm{B}(a, d)$ & $30^{\circ}$ & $0^{\circ}$ & $54^{\circ}$ & 0,809 & 0 & 0,588 & $-0,294$ & 0,866 & 0,405 & 970,5 & 2,44 & 15,70 & $14^{\circ} 7$ & $-25,5$ \\
\hline$C(a, a)$ & $30^{\circ}$ & $0^{\circ}$ & $36^{\circ}$ & 0,588 & 0 & 0,809 & $-0,405$ & 0,866 & 0,294 & 970,5 & 2,44 & 15,70 & $14^{\circ} 7$ & $-25,5$ \\
\hline $\mathrm{D}\left({ }^{d}\right)$ & $11^{\circ}$ & $30^{\circ}$ & $66^{\circ} 6$ & 0,795 & 0,459 & 0,397 & $-0,556$ & 0,812 & 0,175 & 984 & 2,98 & 15,23 & $18^{\circ}$ & -25 \\
\hline$(110) 70(b)$ & $70^{\circ}$ & $45^{\circ}$ & $90^{\circ}$ & 0,707 & 0,707 & 0 & $-0,242$ & 0,242 & 0,940 & 931,3 & 2 & 15,52 & $17^{\circ} 7$ & -26 \\
\hline$(110)$ cyl $24(c)$ & $90^{\circ}$ & $45^{\circ}$ & $24^{\circ}$ & 0,288 & 0,288 & 0,914 & $-0,646$ & $-0,646$ & 0,407 & 1011 & 1,3 & 15 & 0 & -25 \\
\hline (110) cyl 53()$^{(c)}$ & $90^{\circ}$ & $45^{\circ}$ & $53^{\circ}$ & 0,565 & 0,565 & 0,602 & $-0,426$ & $-0,426$ & 0,799 & 1034 & 0,83 & 15 & 0 & -31 \\
\hline
\end{tabular}

(a) Par raison de symétrie les configurations $\mathrm{B}$ et $\mathrm{C}$ sont équivalentes.

(b) Réf. [1] : coupe (110) $-70^{\circ}-$ le présent travail indique plutôt $67^{\circ} 5$.

(c) Réf. [3] : coupe cylindrique $(110)-24^{\circ}$ et $53^{\circ}$.

(d) Cet article.

(e) Angle de dérive (degrés).

(f) Coefficient de température en fréquence du $2^{e}$ ordre $\left(10^{-8} \mathrm{~K}^{-2}\right)$. Ces valeurs ne sont pas très significatives car les CTF du $2^{\mathrm{e}}$ ordre ont été négligés dans les données de départ (annexe IV).

Parmi les points les plus intéressants, on peut noter une coupe $X$ - tournée, qui pour un angle $\varphi=54^{\circ}$ avec l'axe $Z$ et une orientation $\alpha=30^{\circ}$ par rapport à l'axe $Y$ admet un couplage $k^{2}=2,5 \%$ et un coefficient de température nul. Grâce aux symétries, on trouve une configuration équivalente pour l'angle $\varphi=90^{\circ}-54^{\circ}=36^{\circ}$.

Par ailleurs, on a trouvé sur la nappe à coefficient de température en fréquence nul deux configurations possédant un coefficient de couplage électromécanique de $3 \%$. La première configuration est une coupe (110) tournée $\left(\theta=45^{\circ}\right)$, l'axe faisant un angle $\varphi=36^{\circ} 5$ avec $Z$ et dans l'orientation $\alpha=62^{\circ}$. La seconde correspond à $\varphi=66^{\circ} 6, \theta=30^{\circ}$ et $\alpha=11^{\circ}$. Les paramètres caractéristiques de ces coupes sont résumés dans le tableau III.

3. Conclusion. - Le cahier de charges de la plupart des dispositifs impose une tenue en température assez contraignante. Ces exigences sont souvent difficiles à satisfaire dans les dispositifs à ondes élastiques de surface. En outre, un deuxième paramètre important lié à la bande passante nécessaire complique encore le choix des matériaux à utiliser. Cet article montre les limitations des trois principaux matériaux actuellement commercialisés, quartz, niobate de lithium et tantalate de lithium, pour lesquels il n'existe guère de coupe miracle: la double exigence d'un coefficient de température nul et d'un couplage piézo-électrique notable ne peut alors être satisfaite.

L'exploration d'un nouveau matériau, le $\mathrm{Tl}_{3} \mathrm{VS}_{4}$ ouvre toutefois une fenêtre prometteuse puisque celui-ci jouit des deux qualités recherchées. Il faut cependant noter que ce cristal n'est guère encore disponible et qu'en général un délai de plusieurs années est nécessaire à la commercialisation d'un nouveau cristal.

Remerciements. - Une partie des résultats exposés dans cet article a été obtenue grâce au soutien de la Direction des Recherches et Moyens d'Essai.

\section{Bibliographie}

[1] Weinert, R. W. and IsaACS, T. J., « New piezo electric materials which exhibit temperature stability for surface waves ", Proceedings of the 29th Annual Symposium on Frequency Control, 1975, pp. 139-142.

[2] Isaacs, T. J., Gottlieb, M., Daniel, M. R. and FeichtNER, J. D., « $\mathrm{Tl}_{3} \mathrm{VS}_{4}$ as an acousto-optic and surface wave material ». J. Electron. Mater. 4 (1975) 67-75.

13] Jhunjhunwala, A., Vetelino, J. F. and Field, J. C., « Temperature compensated cuts with zero power flow angle in $\mathrm{Tl}_{3} \mathrm{VS}_{4}$ and $\mathrm{Tl}_{3} \mathrm{TaSe}_{4}$ ", Electron. Lett., 12 (1976) 683-684.

. 1] Campbell, J. J. and Jones, W. R., " A method for estimating optimal crystal cuts and propagation directions for excitation of piezo electric surface waves ", IEEE Trans. SU, SU-15 (1968) 209-217.
[5] Feldmann, M., "Analyse des transducteurs à ondes piézo-électriques de surface », Ann. Telecommuni. 28 (1973) 353-370.

[6] Schulz, M. B. and Holland, M. G., « Temperature dependence of surface acoustic wave velocity in lithium tantalate ", IEEE Trans. Son. Ultrason. SU-19 (1972) 381-384.

[7] Slobodnik, A. J. Jr. and Conway, E. D., " New highfrequency high-coupling low-beam-steering cut for acoustic surface waves on $\mathrm{LiNbO}_{3}$ 》 Electron. Lett. 6 (1970) $171-173$

[8] Haines, J. D., Paige, E. G. S., Saunders, A. F. and YounG, A. S., "Simple technique for the accurate determination of delay time variations in acoustic surface wav structures », Electron. Lett. 5 (1969) 678. 
[9] HenafF, J., "Oscillateurs à ondes élastiques de surface ", l'Onde Electrique, 56 no 4 (1976) 189-196.

[10] Smith, R. T. and WeLSH, F. S., "Temperature dependence of the elastic, piezo-electric and dielectric constants of lithium tantalate and lithium niobate ", J. Appl. Phys. 42 (1971) 2219-2230.

\section{ANNEXE I}

\begin{tabular}{|c|c|c|}
\hline & UAFTZ \\
\hline \multicolumn{3}{|c|}{$[\quad(1,1)=+.86740$} \\
\hline \multirow{2}{*}{\multicolumn{2}{|c|}{$=\begin{array}{l}(1,2) \\
(1,3)\end{array}$}} & $=+.699600 \mathrm{E}+10$ \\
\hline & & $=+.119100 \mathrm{E}+11$ \\
\hline$\tau$ & {$[\quad(1,3)$} & $=-.179100 \mathrm{E}+11$ \\
\hline \multicolumn{2}{|c|}{1} & $=+.867466 \mathrm{E}+11$ \\
\hline \multicolumn{2}{|c|}{ 3) } & $=+.119100 \mathrm{E}+11$ \\
\hline \multirow{2}{*}{\multicolumn{2}{|c|}{$(2,4)$}} & $=+.179100 \mathrm{E}+11$ \\
\hline & & $=+.107200 \mathrm{E}+12$ \\
\hline 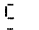 & $(4,4)$ & $=+.579400 \mathrm{E}+11$ \\
\hline E & $(5,5)$ & $=+.579400 \mathrm{~W}+11$ \\
\hline E & $(5,6)$ & $=-.179100 \mathrm{E}+11$ \\
\hline E & $(b, b)$ & $=+.398800 \mathrm{E}+11$ \\
\hline$E$ & $(1,1)$ & $=+.171060 \mathrm{E}+00$ \\
\hline & $(1, \bar{z})$ & $=-.17106 \sigma \mathrm{E}+00$ \\
\hline$E$ & $(1,4)$ & $=-.04 \emptyset 6 \emptyset \emptyset \mathrm{E}+\emptyset \emptyset$ \\
\hline$E$ & $(2,5)$ & $=+.040600 \mathrm{E}+\emptyset 0$ \\
\hline & $(\Sigma, b)$ & $=-.171060 \mathrm{E}+00$ \\
\hline EF & & $=+.450006 \mathrm{E}+01$ \\
\hline EF & $(\Sigma, \bar{L})$ & $=+.450606 \mathrm{E}+01$ \\
\hline EF & & $=+.460006 \mathrm{E}+\not 21$ \\
\hline ET & & $=-.443699 \mathrm{E}-\not) 4$ \\
\hline ET & $(1,2)$ & $=-.299300 \mathrm{E}-0.02$ \\
\hline CT & $(1,3)$ & $=-.495060 \mathrm{E}-63$ \\
\hline ET & $(1,4)$ & $=+.980066 \mathrm{E}-04$ \\
\hline ET & $(\Sigma, \Sigma)$ & $=-.443000 \mathrm{E}-04$ \\
\hline ET & $(2,3)$ & $=-.492000 \mathrm{E}-03$ \\
\hline ET & $(2,4)$ & $=+.980006 \mathrm{E}-0.04$ \\
\hline ET & $(3,3)$ & $=-.189660 \mathrm{E}-63$ \\
\hline$E T$ & & $=-.17 \overline{2} \theta 0 \square \mathrm{E}-0 \overline{3}$ \\
\hline ET & $(5,5)$ & $=-.172000 \mathrm{E}-003$ \\
\hline$[T$ & & $=+.980006 \mathrm{E}-064$ \\
\hline$E T$ & {$[(t, b)$} & $=+.180000 \mathrm{E}-63$ \\
\hline ET & $(1,1)$ & $=-.407000 \mathrm{E}-06$ \\
\hline CT & $(1,2)$ & $=-.724506 \mathrm{E}-05$ \\
\hline ET & $(1,3)$ & $=-.59600 \mathrm{~b}-06$ \\
\hline ET & $(1,4)$ & $=-.130600 \mathrm{E}-07$ \\
\hline ET 2 & $(2,2)$ & $=-.407000 E-066$ \\
\hline ET 2 & & $=-.596000 \mathrm{E}-0 \mathrm{E}$ \\
\hline ET: & & $=-.130000 \mathrm{E}-07$ \\
\hline CT: & 13 & $=-.141200 \mathrm{E}-05$ \\
\hline CT & & $=-.225 \phi \emptyset 6 \mathrm{E}-\emptyset 6$ \\
\hline CT2 & & $=-.2 \angle 5069 \mathrm{E}-06$ \\
\hline E.T. & & $=-.136090 \mathrm{E}-67$ \\
\hline ET: & , sl & $=+.201969 \mathrm{E}-66$ \\
\hline ET 1 & & $=-.1600 \sigma \sigma \mathrm{E}-\not 23$ \\
\hline ET & & $=-.160000 \mathrm{E}-0 \mathrm{O}$ \\
\hline ET: & ,4) & $=-.144 \emptyset \emptyset \sigma E-\emptyset Z$ \\
\hline ET 1 & & $=-.144900 \mathrm{E}-\emptyset \overline{\mathrm{C}}$ \\
\hline ET & $(2,6)$ & \\
\hline F'T : & $(1,1)$ & $=+.280000 \mathrm{E}-04$ \\
\hline F'T 1 & & $=+.280 \emptyset \emptyset \emptyset \mathrm{E}-\emptyset 4$ \\
\hline FT: & $(3,3)$ & $=+.390006 \mathrm{E}-04$ \\
\hline AT: & (1) & $=+.980000 \mathrm{E}-05$ \\
\hline AT & & $=+.980906 \mathrm{E}-65$ \\
\hline AT & (3) & $=+.560000 \mathrm{E}-05$ \\
\hline AT & $(1)$ & $=+.2 \emptyset 4 \emptyset \emptyset \emptyset \mathrm{E}-\emptyset 7$ \\
\hline AT2 2 & & $=+.294060 \mathrm{E}-977$ \\
\hline AT & $(3)$ & $=+.940900 \mathrm{E}-08$ \\
\hline TT T & & 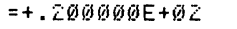 \\
\hline
\end{tabular}

ANNEXe I : Constantes $d u$ quartz utilisées dans le programme de calcul

[Constants of quartz used in the computer program]
ANNEXE II

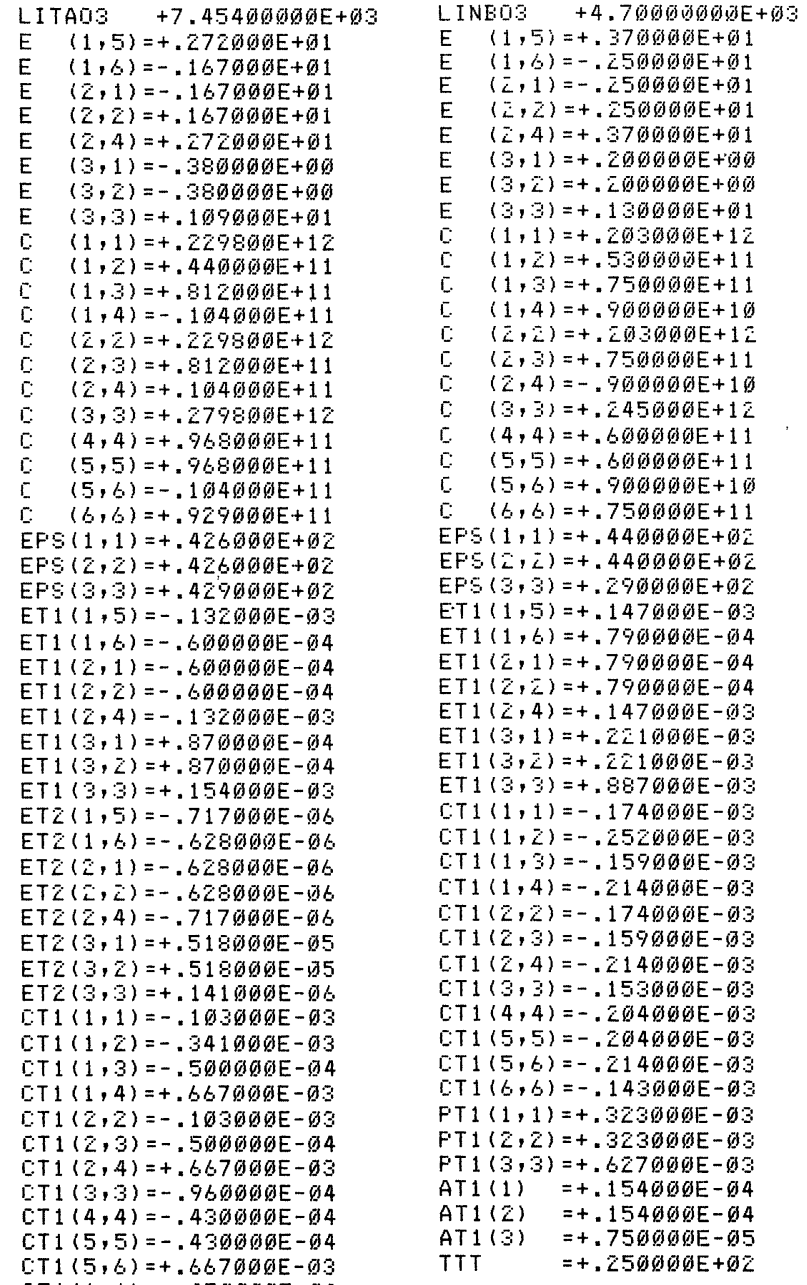

ANNEXE III : Constantes du niobate de lithium [11] [Constants of lithium niobate [11]] CT1 $(6,6)=-.470000 \mathrm{E}-04$ $\operatorname{CTZ}(1,1)=+.779090 \mathrm{E}-97$ $\operatorname{CTZ}(1,2)=-.118000 \mathrm{E}-96$ $\operatorname{CT} 2(1,3)=+.600006 \mathrm{E}-06$ $\operatorname{cT} 2(1,4)=+.167000 \mathrm{E}-05$ CTZ $(2, Z)=+.770000 \mathrm{E}-07$ CT $Z(Z, 3)=+.699696 \mathrm{E}-96$ $\operatorname{CTZ}(2,4)=+.167 \emptyset \emptyset \emptyset \mathrm{E}-05$

\section{ANNEXe IV}

TL3VS4+6.14000000E+03

[: $\quad(1,1)=+.484000 \mathrm{E}+1$

c) $(Z, Z)=+.484000 \mathrm{E}+11$

c) $(3,3)=+.484090 \mathrm{E}+11$

c. $\quad(1, \overline{2})=+.165000 \mathrm{E}+1$

c. $(2,3)=+.165000 \mathrm{E}+11$

c) $(4,4)=+.470000 \mathrm{E}+10$

c $\quad(5,5)=+.470009 \mathrm{E}+10$

c. $(b, t)=+.470000 \mathrm{E}+10$

E $\quad(1,4)=+.350600 \mathrm{E}+00$

E $\quad(2,5)=+.350000 E+00$

E $\quad(3,6)=+.350000 E+00$

$\operatorname{EF} S(\bar{L}, \bar{z})=+.140000 \mathrm{E}+0 \bar{z}$

$\operatorname{EFS}(3,3)=+.140000 \mathrm{E}+02$

CT $1(1,1)=-.572000 \mathrm{E}-09$

CT $1(2,2)=-.572006 \mathrm{E}-09$

CT $1(3,3)=-.572000 \mathrm{E}-03$

CT $1(1,2)=-.210000 \mathrm{E}-003$

CT $1(1,3)=-.210000 \mathrm{E}-03$

CT1 $(2,3)=-.210000 \mathrm{E}-03$

CT $1(4,4)=+.101000 \mathrm{E}-03$

CT1 $(5,5)=+.101000 \mathrm{E}-0 \mathrm{~S}$ CT1 $16, b)=+.161000 \mathrm{E}-03$ CT2 $(5,5)=-.570000 \mathrm{E}-06$ CTC $(6,6)=-.570000 \mathrm{E}-06$ AT $1(Z)=+.254000 \mathrm{E}-04$ AT1 13$)=+.254000 \mathrm{E}-04$ TTT $=+.250699 \mathrm{E}+\emptyset \mathrm{C}$

ANNEXE IV : Constantes $d u \mathrm{Tl}_{3} \mathrm{VS}_{4}$ [1]

[Constants of $\mathrm{Tl}_{3} \mathrm{VS}_{4}$ [1]]
[. $\quad(1,3)=+.165000 \mathrm{E}+1$

EPG $(1,1)=+.140000 \mathrm{E}+02$

CT $(4,4)=+.101000 \mathrm{E}-63$ CT2 $(4,4)=-.579090 \mathrm{E}-06$ AT $1(1)=+.254000 \mathrm{E}-94$ $\operatorname{CT} 2(3,3)=-.321090 \mathrm{E}-06$ CTZ $(4,4)=+.167969 \mathrm{E}-66$ $\mathrm{CT} Z(5,5)=+.167090 \mathrm{E}-96$ $\operatorname{CT2}(5,6)=+.167000 \mathrm{E}-05$ CT2 $(6,6)=+.124000 \mathrm{E}-06$ CT $(1,1)=+.124000 \mathrm{E}-06$ PT $1(2,12)=+.329000 \mathrm{E}-03$ PT1 12,2$)=+.329000 \mathrm{E}-03$ PT $1(3,3)=+.116000 \mathrm{E}-\emptyset 2$
FTZ $(1,1)=+.428000 \mathrm{E}-06$ $F^{\top} T Z(Z, Z)=+.4280 \emptyset \emptyset E-\emptyset b$ $\operatorname{PT} Z(3,3)=+.780009 \mathrm{E}-05$ AT $1(1)=+.161000 \mathrm{E}-04$ AT1 (2) $=+.161000 \mathrm{E}-04$ AT $1(3)=+.410000 \mathrm{E}-05$ AT $1(3)$ AT $2(1)=+.790006 \mathrm{E}-98$ ATZ $(Z)=+.700900 \mathrm{E}-00$ $\begin{array}{ll}\text { ATZ }(3) & =-.190090 \mathrm{E}-07 \\ \text { TTT } & =+.250000 \mathrm{E}+0 \mathrm{C}\end{array}$

AnNEXE II : Constantes $d u$ tantalate de lithium [10] [Constants of lithium tantalate [10]] 